\title{
Antenatal microbial colonisation of mammalian gut
}

Elisa Borghi ${ }^{1 \S^{*}}$, Valentina Massa ${ }^{1 \S}$, Marco Severgnini ${ }^{2}$, Grazia Fazio $^{3}$, Laura Avagliano ${ }^{1}$, Elena Menegola $^{4}$, Gaetano Bulfamante ${ }^{1}$, Giulia Morace ${ }^{1}$, Francesca Borgo ${ }^{1}$

${ }^{1}$ Dipartimento di Scienze della Salute, Università degli Studi di Milano, 20142 Milan, Italy;

${ }^{2}$ Institute of Biomedical Technologies, National Research Council, 20090 Segrate, Italy;

${ }^{3}$ Centro Ricerca Tettamanti, Clinica Pediatrica, Dipartimento di Medicina e Chirurgia, Università degli Studi di Milano-Bicocca, Fondazione MBBM, Monza, Italy

${ }^{4}$ Dipartimento di Scienze e Politiche Ambientali, Università degli Studi di Milano, 20133 Milan, Italy;

$\S$ These authors equally contributed to this work

*corresponding author: elisa.borghi@unimi.it

Running title: Mammals born as super-organisms 


\section{ABSTRACT}

The widely accepted dogma of intrauterine sterility and initial colonisation of the newborn during birth has been blurred by recent observations of microbial presence in meconium, placenta and amniotic fluid. Given the importance of a maternal-derived in utero infant seeding, it is crucial to exclude potential environmental or procedural contaminations, and to assess foetal colonisation before parturition. To ascertain antenatal microbial colonisation in mammals, we analysed sterilely collected intestinal tissues from rodent foetuses in parallel with experimental controls, and tissues from autoptic human foetuses. Next generation sequencing (NGS) showed the presence of pioneer microbes in both rat and human intestines, as well as in rodent placentas and amniotic fluids. Live microbes were isolated from culture-dependent analyses from homogenized rat foetal intestines.

Microbial communities showed foetus- and dam- dependent clustering, confirming the high interindividual variability of microbiota even in the antenatal period. Fluorescent in situ hybridisation analysis confirmed the microbes' existence in the lumen of the developing gut.

These findings have vast implications for an emerging field of enhancing the management of healthy pregnancies, and for understanding how the infant microbiome starts and it is thus shaped. 


\section{INTRODUCTION}

Foetus, amniotic fluid and chorioamnion tissue have long been considered sterile until birth or rupture of the amniotic sac. However, recent evidence shows that the intrauterine sac environment harbours a diversity of microorganisms even in physiological pregnancies [1-3], contradicting the long-standing dogma of "womb sterility" [4]. Indeed, the cultivable genera Enterococcus, Streptococcus, Staphylococcus, and Propionibacterium have been isolated from umbilical cord blood of healthy neonates born by caesarean section [5]. Within the abundant literature on human placenta microbiome composition [3,6,7], a recent study combining $16 \mathrm{~S}$ ribosomal DNA-based and whole-genome shotgun metagenomic analyses, showed the presence of a unique placental microbiota, strongly resembling the maternal oral bacteria, with the dominance of Firmicutes, Tenericutes, Proteobacteria, Bacteroidetes, and Fusobacteria phyla [3]. In addition, amniotic fluid and placenta were found to have similar microbial communities, consistent across individuals [6]. Lactic acid bacteria and enteric bacteria have been reported in meconium collected after birth [8], and a certain degree of similarity has been demonstrated between meconium and amniotic fluid [6], probably related to liquid swallowing by the foetus during pregnancy.

All these data suggest but not prove that humans might come in to contact with bacteria before birth, and, depending on the time of gestation and the type of bacteria that first seed the foetus, this antenatal colonization might have important physiological and clinical consequences. Indeed, microbes, either true pioneer or transient species, could expose the developing foetus to a diverse array of antigens [9] that educate the foetal immune system toward tolerance and participate in the full development of the gut-associated lymphoid tissue [10].

At the same time, toxins and viable microorganisms, through active and passive transport from maternal circulation to the placenta could gain direct entry into foetal circulation, eliciting infective and/or inflammatory processes [11].

Despite these recent advances in the field, a conclusive analysis of antenatal microbial colonisation has not been reported [12], leaving a gap in the knowledge of this important developmental process. The present study is aimed at ascertaining antenatal microbial 
colonisation of mammalian foetal intestinal tissues. Due to the vast medical and scientific consequences of a "colonised womb", it is crucial to exclude potential environmental or procedural contamination. To address this issue, a rodent animal model was used to allow sterile experimental conditions. This was compared to human gut samples from foetal autopsies that were studied using a 16S rRNA amplicon-based NGS approach, validated by in situ detection.

\section{RESULTS}

\section{Microbial species are identifiable and viable in rodent foetuses in utero}

We collected, under sterile conditions as described in the method section, intestine, placenta and amniotic fluid from five rat foetuses: three foetuses (numbered 1-3) from one dam (dam A) and two (numbered 4-5) from the other (dam B). The tissues were analysed by $16 \mathrm{~S}$ rRNA sequencing.

An average of 259,465 reads were obtained per sample, giving a total of $4,670,364$ reads overall. Paired-end reads generated from the original DNA fragments using Illumina MiSeq Next Generation Sequencing were merged and quality-filtered producing a total of 1,560,296 sequence tags from the gut samples, and 982,017 and 900,070 from placentas and amniotic fluids, respectively.

9 different bacterial phyla were identified in rat foetal samples following negative control subtraction. The most represented phyla (Fig. 1A), using a cut-off applied of a relative abundance greater than $1 \%$ in at least one experimental group, were Firmicutes (mean relative abundance \pm

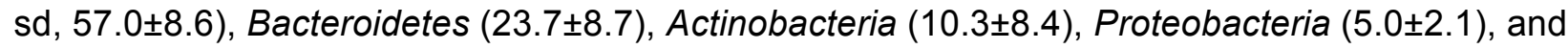
Verrucomicrobia $(2.8 \pm 1.9)$.

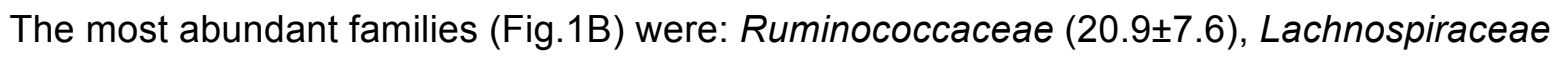

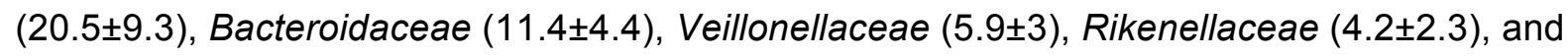

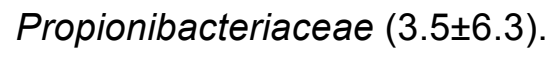

To assess whether identified bacteria were viable, two different experiments were performed using a pool of three dissected foetal intestines, sterilely collected and stored at $-80^{\circ} \mathrm{C}$. Following a growth enrichment step, Gram stain was performed and few dispersed bacteria were detected. In 
the second experiment, we performed a further step of enrichment in anaerobic blood culture bottle prior subculturing. The bottle turned out positive on day 4, revealing the presence of live microbes. Colony picking and MALDI-TOF processing on the second experiment cultured bacteria allowed for the identification of Propionibacterium spp..

\section{Microbial community is characteristic of foetuses and dams}

In order to understand the main determinants constituting the microbial diversity, we evaluated the differences amongst the samples on both richness and composition. Tissues (i.e. intestine, placenta, and amniotic fluid), dams (i.e.: A and B) and foetuses were considered for microbiota profiling.

The analysis of samples biodiversity ( $\alpha$-diversity) showed clustering according to dam and foetus rather than analysed tissue. Faith's phylogenetic diversity (Fig. 2A), measured based on distances, and observed species metrics showed a significant separation dependent on foetus (Mann-Whitney test: $p=0.016$ and 0.019 , respectively). Intra- and inter-group unweighted Unifrac distances confirmed foetus-dependent microbial profiles ( $p=0.01$, Fig. $2 B$ ). Both the metrics showed a separation dependent also on dam (permutation based test: $p=0.004$ and 0.036 , respectively). Interestingly, no significant separation was observed based on tissue type $(p>0.05)$, independently from the metric used to compare distributions.

To evaluate whether different samples were characterized by distinct microbiota composition profiles ( $\beta$-diversity, Fig. $3 \mathrm{~A}$ ), the distribution of Unifrac distances was assessed. As with the $\alpha$-diversity, the $\beta$-diversity analyses clustered according to foetus (adonis test $p=0.009$ and 0.006 on unweighted and weighted Unifrac distances, respectively) and to dam (adonis test $p=0.015$ on weighted Unifrac distances).

Distributions of unweighted Unifrac distances (Fig. 3B) on foetus were statistically different $(p=0.01)$, whereas weighted Unifrac distances were not $(p=0.09)$, indicating that significant differences are present in sub-dominant components of the microbiota. In detail, foetus 1 was characterized by a high relative abundance of Propionibacteriaceae ( $14.3 \%$ compared to an average of $1.1 \%$ in other foetuses) and Corynebacteriaceae $(4.1 \%$ compared to an average of 
$0.4 \%$ in other foetuses). Foetus 3 showed an increased abundance of Erysipelotrichaceae (1.7\% compared to an average of $0.9 \%$ in other foetuses), and foetus 5 was enriched in Porphyromonadaceae (average relative abundance $2.4 \%$ compared to $1.0 \%$ in other foetuses) (Supplementary Fig. 1 and Supplementary Fig. 2A).

Analysis of distances based on sample origin (i.e. foetus, tissue and dam) showed a trend indicating similarity in microbial profiles among foetuses (Fig. 3C).

Analysis of observed species metric $(p=0.036)$ and Faith's phylogenetic distances $(p=0.004)$ allowed for a significant separation among dams, with dam B presenting with a lower biodiversity (Supplementary Fig. 3).

The microbiota signature for each tissue appeared less distinct, with only some hints of a lower presence of Verrucomicrobiaceae in placenta tissue (relative abundance: $1.1 \%$ compared to $2.7 \%$ and $3.7 \%$ in amniotic fluid and intestine, respectively) and a trend of higher relative abundance of Barnesiellaceae in the intestine (4.0\% compared to $1.4 \%$ and $1.5 \%$ in placenta and amniotic liquid, respectively) (Supplementary Fig. 2C and Supplementary Fig. 4). Few differences emerged in the comparison between dam A and B (Supplementary Fig. 2B and Supplementary Fig. $5)$.

\section{Bacteria are visualised in the gut during rodent foetal development}

To assess bacteria distribution, in situ analysis was performed on whole sectioned foetuses. Fluorescent detection revealed the presence of bacteria in the gut lumen of developing rat foetuses (Fig. 4A and 4B). In particular, eubacteria (green fluorescence) could be visualised on the different analysed sections, confirming that bacteria colonize the rodent intestine before birth. Probe for Staphyloccaceae did not give positive fluorescent signal in any of the tissue sections analysed.

\section{Bacteria are present in the gut during human foetal development}

Paraffin-embedded intestinal tissues from three third trimester (gestational age 29, 31 and 33 weeks) human foetuses were screened for the presence of foetal microbiota. 
In all analysed samples, bacteria were observed. The most represented phyla (Fig. 5A)

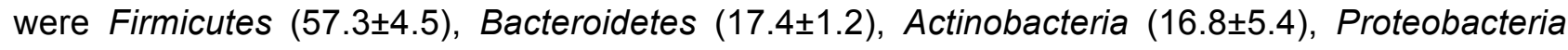
(4.9 \pm 2.2$)$, and Verrucomicrobia $(2.7 \pm 1.7)$. At family level, the most abundant taxa were:

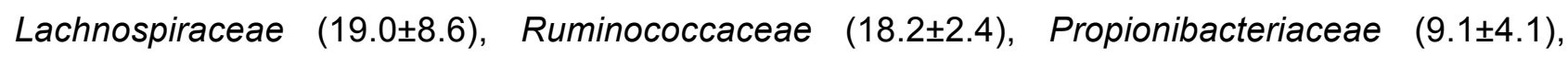

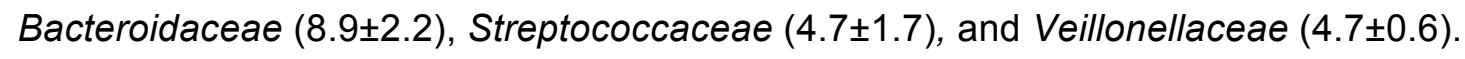

The presence of bacteria indicated by the data obtained by NGS analysis was further validated by visualising eubacteria in the lumen of the developing gut by fluorescent in situ hybridization (Fig. $5 B)$.

\section{DISCUSSION}

Commensal microorganisms have coevolved with their natural hosts giving rise to holobionts (host plus symbiotic microbes) [13]. Microbes-encoded genes fully support and integrate the host genome in a comprehensive gene system that participates in holobiont phenotype establishment [14] and obey to fundamental genetic rules such as maternal transmission $[4,15]$ and natural selection. Indeed, microbiota adaptation has been observed in different human niches during pregnancy [16].

Two recent papers $[17,18]$ suggest a direct effect of maternal microbiome-immune system interaction on offspring neurodevelopment. In addition, the presence of a meconium microbiota strongly supports the existence of maternal microbial transmission in utero [8].

In this study, for the first time to our knowledge, we show that bacteria are present in anatomical foetal mammalian gut sections. Importantly, some species were found to be viable following specific culturing conditions. Given the limited dimension of the rat foetal intestine and the consequent paucity of the sample, culture requires subsequent steps of enrichment inevitably leading to a biased selection and a underestimation of members of the microbial community.

Reported relative abundance in meconium $[6,8,19]$ indicate a consistent presence of Proteobacteria, whereas in this study, both in human and rat developing gut, Firmicutes were found to be more represented. These changes in microbiota may be a consequence of physiological changes that occur during birth. 
Supporting the important role of the microbiota community in the developing mammalian gut, the phyla composition found in analysed samples (Firmicutes, Bacteroidetes, Actinobacteria, Proteobacteria and Verrucomicrobia) closely resembles those reported in healthy adult human gut $[20,21]$. It is important to note that, compared to adult and childhood tissues [22,23], the developing gut is enriched in Actinobacteria, and depleted in Bacteroidetes. Actinobacteria abundance has been shown to progressively increase in infant faeces during lactation and to then decrease when solid food is introduced in the diet. The opposite has been reported for Bacteroidetes [24-26]. Hence, the difference between foetal and adult gut composition is in line with infant faecal microbiota, suggesting that solid food might be responsible for the switch. It could also suggest that the presence of a maternally provided reservoir of bifidobacteria, that with human milk oligosaccharides are known to be fundamental for the development of a balanced infant microbiota and a fully functional gastrointestinal tract [24,27-29].

Another difference found in our study compared to bacteria composition reported in meconium collected after birth $[6,8]$, is that Staphylococcaceae and Streptococcaceae appear to be less abundant during uterine life. It is known that staphylococci are characteristic of higher respiratory tract and skin microbiota, hence it is conceivable that colonisation occurs during birth or from the first days of life through contact with maternal tissues. It has also recently been shown that both genera are abundant in colostrum and maternal milk [30], indicating a possible dual colonising path.

Analysis of $16 \mathrm{~S}$ rRNA amplicon data clearly showed that microbiota composition is foetus- and dam- specific rather than tissue specific. Indeed, bacterial families found in amniotic fluid and placenta overlap with those found in the corresponding foetus. Importantly, the specificity seems to be independent of growing environment (i.e., uterine tissues etc.) but it seems to relate to micro niches (i.e. embryonic implant). This is true not only for the microbial community, but also for other developmental determinants, such as genetic, metabolic, biochemical or epigenetic components known to be specific to the single developing organism and not always shared among all siblings. This is in addition to the possible confounding factors of gender, time and site of implantation etc. 
Given that bacteria colonise mammalian gut during intrauterine life, the fundamental question remains as to the source and the path of this in utero seeding or exposure. Recent findings [3] have described how, analysing a significant number of placentas collected after birth, the placental microbiota shares more similarities with that of the oral composition compared to vaginal, skin and/or gut communities. Rather than identifying the origin of placental microbiota, these data support the exclusion of a passive dispersion through excreting organs. In the literature, a possible microbiota colonisation in utero has been often hypothesised as a consequence of pathogens known to be able to reach the developing foetus. Our data suggest an alternative mechanism, where pathogens may pass the maternal barrier as a consequence of the necessary permissiveness to commensal bacteria [4], instead of resulting from infectious events (reviewed in Doran et al. [31])

Clearly, considering the accumulating evidence for a strict relationship between microbiome and health status, in all studied settings (age, gender, ethnicity, mtDNA SNP, haplogroup, etc.) [32-37] and the importance of maintaining or replenishing the microbial community in pathological conditions $[35,38,39]$, the present paper opens an enormous field of investigation relating to the management of healthy pregnancies [40]. To pave this way, basic questions should first be addressed, as when colonisation occurs and through which routes, and improvement in culturomics might in the future allow for establishing in vitro foetal microbiota studies [41].

In conclusion, through detecting bacteria in rat and human developing gut both by Next Generation Sequencing and by in situ visualisation, we provide evidence that mammals do not, as often postulated, grow to be super-organisms [42-44], but instead they are born as such.

\section{METHODS}

\section{Animals and housing}

CD albino rats (Sprague-Dawley) were maintained in standard conditions (light 6 a.m.-6p.m., $\mathrm{T}=22+/-2^{\circ} \mathrm{C}$, humidity $=55+/-5 \%$ ) with tap water and food (Mucedola standard diet) ad libitum. Virgin females were caged overnight with males of proven fertility. The day of positive vaginal smear was considered as day 0 . The pregnant rats were housed individually. Animals were 
euthanized by carbon dioxide inhalation and bilaterally pregnant uterine samples were collected in the morning of gestational day 16. Amniotic fluid, placentae and foetal intestines were dissected, collected in sterile conditions, and stored at $-80^{\circ} \mathrm{C}$ until use. For dissection, uteruses were placed in sterile saline solution under laminar flow cabinet, and all procedures were conducted in the hood using UV sterilised equipment within a sterile field created by a Bunsen burner, until samples were placed in sterile tubes. For in situ hybridisation, whole foetuses were collected in paraformaldehyde $4 \%(\mathrm{v} / \mathrm{v})$, and kept at $4^{\circ} \mathrm{C}$ in rotation for 4 days. Samples were then washed twice in phosphate saline solution (PBS), rehydrated through a graded series of alcohols, and paraffin-embedded.

All animal procedures were conducted in accordance with the ethical guidelines approved by the University of Milan in compliance with national (Dlgs 26/2014) and international laws and policies (EEC Council Directive 86/609).

\section{Human samples}

From a database of foetal autopsies performed after legal termination of pregnancy, we selected, among cases without malformations and with normal karyotype, three random foetuses on the basis of gestational age, and of gut tissue availability. $4 \mu \mathrm{m}$ thick tissue sections from formalinfixed, paraffin-embedded tissue's samples were cut and processed for in situ hybridization by deparaffinisation in xylene and rehydration through a graded series of alcohols. For NGS analysis, tissue sections, cut under laminar flow cabinet using sterile blades and placed in sterile tubes, were washed twice with $1 \mathrm{~mL}$ of Histo-Clear (Sigma Aldrich, Milan, Italy) for 15 minutes with rotation at $56^{\circ} \mathrm{C}$ or until diaphanisation. Tissue was recovered by centrifugation, washed in ethanol and dried for DNA extraction.

The study was exempt from Institutional Review Board approval because, following Italian Data Protection Act 9/2013, autopsy material sampled for diagnostic purposes can be used for research as long as patient privacy is ensured. This law is in line with European Commission recommendation $\mathrm{n}$. $\operatorname{Rec}(2006) 4$. 


\section{Next Generation Sequencing analysis}

Total bacterial DNA extraction was performed using the QIAamp DNA Microbiome Kit (QIAGEN, Hilden, Germany), following manufacturer's instructions. Particular attention was paid to avoid environmental contamination of collected samples, and cross-contamination between samples. Samples were individually processed for DNA extraction under laminar flow cabinet, following UV sterilisation.

16S rRNA gene amplicon libraries were performed with a two-step barcoding approach according to Illumina 16S Metagenomic Sequencing Library Preparation (Illumina, San Diego, CA, USA), which amplifies two hypervariable regions (i.e.: V3, V4) of the $16 \mathrm{~S}$ rRNA bacterial gene. Library concentration and exact product size were measured using a KAPA Library Quantification Kit (Kapa Biosystems, Woburn, MA, USA) and Agilent 2100 Bioanalyzer System (Agilent, Santa Clara, CA, USA), respectively. Agilent analysis for evaluating the correct predicted size of amplicons showed no bands in negative controls, extracted and processed in parallel with samples (Supplementary Fig. 6). Prior to sequence, libraries were pooled using Amicon Ultra 0.5ml Centrifugal Filters (Merck Millipore Ltd, Tullagreen, Carrigtwohill Co, Cork, Ireland).

The resulting library was loaded on a MiSeq ${ }^{\circledR} 500$ cycle-v2 cartridge to obtain a paired-end $2 \times 250$ bp sequencing. Demultiplexed FASTQ files were generated by Illumina MiSeq Reporter and 2.5 Gbases were obtained.

\section{Fluorescence in situ hybridization (FISH) analysis}

Paraffin-embedded tissue specimens ( $4 \mu \mathrm{m}$ thick) were deparaffinised by sequential steps in xylene. Then samples were re-hydrated in $95 \%$ ethanol, $90 \%$ ethanol, and finally deionized water. The slides were air-dried prior to hybridization.

FISH probe sequences for Eubacteria (EUB 338-I, 5'-GCTGCCTCCCGTAGGAGT-3'; EUB 338-III, 5'-GCTGCCACCCGTAGGTGT-3'), encompassing all bacterial species in Bacteria domain (labelled with FITC), Bacteroides (BAC303, 5'-TCCTCCATATCTCTGCGC-3', Cy3), and Lachnospiraceae (LACHNO, 5'-TTCCCATCTTTCTTGCTGGC-3', Cy5) were obtained from probeBase website [45]. Negative control probe (complementary to EUB 338-I probe, NON-EUB, 
5'-ACTCCTACGGGAGGCAGC-3') was also hybridised to evaluate non-specific binding.

Staphylococcaceae probe (STAPHY, 5'-TCCTCCATATCTCTGCGC-3', Cy3) sequence was designed as described by Gey et al. [46], and used to assess possible environmental contaminations in sampling. Probes were purchased from Integrated DNA Technologies (IDT, San Jose, CA, USA). Hybridisation was carried out using standard methods $[46,47]$. Briefly, sections were deparaffinised and rehydrated in serial solutions. Following section air-drying, specific oligonucleotide probes were hybridised using conditions optimized for each probe for stringent hybridisations: BAC 303 at $48^{\circ} \mathrm{C}$ and $10 \%$ formamide; STAPHY, and LACHNO at $48^{\circ} \mathrm{C}$ and $30 \%$ formamide; EUB $338-I$, and EUB $338-\mathrm{III}$, at $48^{\circ} \mathrm{C}$ and $10 \%$ or $30 \%$ formamide according to the paired probes.

DAPI (4',6-diamidino-2-phenylindole) counterstaining was applied to assess prokaryotic and eukaryotic nuclear morphology.

In this set of experiments, a number of controls were used: sections hybridised with STAPHY probe, that resulted negative, to exclude common contaminants; sections hybridised with NONEUB probe, as negative control; artificially contaminated sections hybridised with STAPHY probe, that resulted positive, as technical control.

Images of probe-labelled sections were acquired using a confocal laser scanning microscope (CLSM, TCS SP2, Leica, Wetzlar, Germany). Microorganisms were checked for position, size and morphology. Confocal images were acquired by series and sequential scan mode. Photomultiplier tube detectors were adjusted to minimise the bleed-through of fluorescent emissions and to optimise signal/noise ratio, in particular versus tissue auto fluorescence.

\section{Culturing of rodent intestine samples}

A pool of dissected foetal intestines, previously frozen at $-80^{\circ} \mathrm{C}$, were homogenised in $1 \mathrm{~mL}$ of brain-heart infusion (BHI, supplemented with L-cysteine and $1 \mathrm{pg} / \mathrm{ml}$ rezasurin) broth and incubated for 3 days at $37^{\circ} \mathrm{C}$ in anaerobic conditions by using an anaerobic jar and an atmosphere generator (BD Diagnostics, Heidelberg, Germany). Following the pre-incubation step, Gram staining was performed.To further promote the growth of anaerobic bacteria, we inoculated the 
pre-incubated broth in an anaerobic blood culture bottle (BD Bactec Plus Lytic/10 Anaerobic, Germany). Finally a subculture step, using both microaerophilic and anaerobic conditions, was performed on Columbia CNA agar, TSA II + 5\% Sheep Blood, MacConkey, chocolate and BHI agar. Colony identification was performed, after $48 \mathrm{~h}$ incubation, using mass spectrometry (MALDITOF MS, Biomérieux).

\section{Data analysis}

Sequencing reads were processed, filtered and analysed following similar procedures described in Borghi et al. [48]. Briefly, read pairs were merged together by PandaSeq software [49] discarding fragments of length $<300$ bases or $>900$ bases, as well as non-overlapping sequences. Then, fragments were quality-filtered, clustered into OTUs (Operational Taxonomic Units) and taxonomically classified against the 13.8 release of the Greengenes bacterial 16 S rRNA database (http://greengenes.lbl.gov) using the QIIME suite (v. 1.8.0) [50].

Biodiversity ( $\alpha$-diversity) was evaluated by permutation-based t-tests, whereas "adonis" of the R package "vegan" was used for bacterial composition ( $\beta$-diversity). In addition, due to the reduced number of samples per category, we devised an alternative strategy for comparing the distributions of distances within and between each experimental group for both $\alpha$-diversity and $\beta$ diversity evaluations. Each sample was assigned to an experimental group according to one of the associated labels (i.e.: tissue type, dam or foetus number); then, a distance between each sample and all the others was calculated. This allowed distinguishing distances between samples belonging to the same ("intra-category" distance) or to a different ("inter-category" distance) experimental group. This strategy was applied for evaluating the absolute difference for a-diversity indexes (i.e.: chao1, Shannon index, observed species and Faith's phylogenetic distance) and the weighted or unweighted Unifrac distances [51] ( $\beta$-diversity). A Mann-Whitney U-test was applied for comparing the distributions of "intra-" and "inter-category" distances.

Details of statistical methods are provided in Supplementary information. 
Data availability. Raw sequence data determined in this study are available at NCBI Short Read Archive (SRA, https://www.ncbi.nlm.nih.gov/sra/) under Accession numbers PRJNA379373 and PRJNA379370.

ACKNOWLEDGMENTS: This study was funded by a Research Grant 2016 of the European Society of Clinical Microbiology and Infectious Diseases (ESCMID) to F.B, and by Università degli Studi di Milano. We thank Raffaella Adami for her assistance in confocal laser microscopy techniques, Francesca Di Renzo, Silvia Rigamonti, and Caterina Biassoni for technical assistance. We are grateful to Ms Dawn Savery, Dr Jon Wilson and Dr Nicola Segata for commenting on the manuscript.

AUTHOR CONTRIBUTIONS: E.B., V.M. and F.B. conceived and designed the study; E.M and V.M. performed rodent housing and sampling; L.A. and G.B. performed human tissue sampling; E.B. F.B. and G.F. performed experiments; M.S. performed bioinformatics analysis; M.S., E.B. and F.B. analysed the data; V.M. and E.B. wrote the paper. All authors made comments on the manuscript; G.M. supervised the project.

COMPETING FINANCIAL INTERESTS: The authors declare no conflict of interest.

\section{REFERENCES}

1. Brugman, S., Perdijk, O., van Neerven, R. J. J. \& Savelkoul, H. F. J. Mucosal Immune Development in Early Life: Setting the Stage. Arch Immunol Ther Exp 2015;63: 251-268.

2. Ardissone AN, de la Cruz DM, Davis-Richardson AG, Rechcigl KT, Li N, Drew JC, et al. Meconium microbiome analysis identifies bacteria correlated with premature birth. PLoS One. 2014;9(3): e90784.

3. Aagaard K, Ma J, Antony KM, Ganu R, Petrosino J, Versalovic J. The placenta harbors a unique microbiome. Sci. Transl. Med. 2014;6: 237ra65. 
4. Funkhouser LJ, Bordenstein SR. Mom Knows Best: The Universality of Maternal Microbial Transmission. PLoS Biol. 2013;11:1-9.

5. Jiménez E, Fernández L, Marín ML, Martín R, Odriozola JM, Nueno-Palop C et al. Isolation of commensal bacteria from umbilical cord blood of healthy neonates born by cesarean section. Curr. Microbiol. 2005;51: 270-274.

6. Collado MC, Rautava S, Aakko J, Isolauri E, Salminen S. Human gut colonisation may be initiated in utero by distinct microbial communities in the placenta and amniotic fluid. Sci. Rep. 2016; 6: 23129.

7. Prince AL, Ma J, Kannan PS, Alvarez M, Gisslen T, Harris RA et al. The placental membrane microbiome is altered among subjects with spontaneous preterm birth with and without chorioamnionitis. Am. J. Obstet. Gynecol. 2016; 214: 627e1-627e16.

8. Jiménez E, Marín ML, Martín R, Odriozola JM, Olivares $\mathrm{M}$, Xaus $\mathrm{J}$ et al. Is meconium from healthy newborns actually sterile? Res. Microbiol. 2008; 159: 187-193.

9. Avagliano L, Marconi AM, Candiani M, Barbera A, Bulfamante, G. Thrombosis of the umbilical vessels revisited. An observational study of 317 consecutive autopsies at a single institution. Hum. Pathol. 2010; 41: 971-979.

10. Belkaid Y, Hand TW. Role of the microbiota in immunity and inflammation. Cell. 2014; 157: 121-141.

11. Fardini Y, Chung P, Dumm R, Joshi N, Han YW. Transmission of diverse oral bacteria to murine placenta: Evidence for the oral microbiome as a potential source of intrauterine infection. Infect. Immun. 2010;78: 789-1796.

12. Perez-Muñoz ME, Arrieta M-C, Ramer-Tait AE, Walter J. A critical assessment of the 'sterile womb' and 'in utero colonization' hypotheses: implications for research on the pioneer infant microbiome. Microbiome 2017;5: 48.

13. Brucker RM, Bordenstein SR. Speciation by symbiosis. Trends Ecol Evol. 2012; 27: 443-451.

14. Bordenstein SR, Theis KR. Host Biology in Light of the Microbiome: Ten Principles of Holobionts and Hologenomes. PLoS Biol. 2015;13: e1002226.

15. Asnicar F, Manara S, Zolfo M, Truong DT, Scholz M, Armanini F, Ferretti P, Gorfer V, Pedrotti A, Tett A, Segata N. Studying Vertical Microbiome Transmission from Mothers to Infants by Strain-Level Metagenomic Profiling. mSystems. 2017; 17;2(1). pii: e00164-16.

16. Nuriel-Ohayon M, Neuman H, Koren O. Microbial Changes during Pregnancy, Birth, and Infancy. Front Microbiol. 2016;7: 1031.

17. Shin Yim Y, Park A, Berrios J, Lafourcade M, Pascual LM, Soares N, Yeon Kim J, Kim S, Kim H, 
Waisman A, Littman DR, Wickersham IR, Harnett MT, Huh JR, Choi GB. Reversing behavioural abnormalities in mice exposed to maternal inflammation. Nature. 2017;549: 482-487.

18. Kim S, Kim H, Yim YS, Ha S, Atarashi K, Tan TG, Longman RS, Honda K, Littman DR, Choi GB, Huh JR. Maternal gut bacteria promote neurodevelopmental abnormalities in mouse offspring. Nature. 2017;549: 528-532.

19. Chu DM, Ma J, Prince AL, Antony KM, Seferovic MD, Aagaard KM. Maturation of the infant microbiome community structure and function across multiple body sites and in relation to mode of delivery. Nat. Med. 2017;23(3): 314-326.

20. Huttenhower C, Human Microbiome Project Consortium. Structure, function and diversity of the healthy human microbiome. Nature 2012;486: 207-214.

21. Qin J, Li R, Raes J, Arumugam M, Burgdorf KS, Manichanh C et al. A human gut microbial gene catalogue established by metagenomic sequencing. Nature. 2010; 464(7285): 59-65.

22. Greenhalgh K, Meyer KM, Aagaard KM, Wilmes P. The human gut microbiome in health: establishment and resilience of microbiota over a lifetime. Environ. Microbiol. 2016;18: 2103-2116.

23. Riva A, Borgo F, Lassandro C, Verduci E, Morace G, Borghi E, Berry D. Pediatric obesity is associated with an altered gut microbiota and discordant shifts in Firmicutes populations. Environ Microbiol. 2017;19(1): 95-105.

24. Turroni F, Ribbera A, Foroni E, van Sinderen D, Ventura M. Human gut microbiota and bifidobacteria: From composition to functionality. Antonie van Leeuwenhoek. 2008;94: 35-50.

25. Scott KP, Gratz SW, Sheridan PO, Flint HJ, Duncan SH. The influence of diet on the gut microbiota. Pharmacol. Res. 2013;69: 52-60.

26. Koenig JE, Spor A, Scalfone N, Fricker AD, Stombaugh J, Knight R et al. Succession of microbial consortia in the developing infant gut microbiome. Proc. Natl. Acad. Sci. U. S. A. 2011;108 Suppl: 4578-4585.

27. Asakuma S, Hatakeyama E, Urashima T, Yoshida E, Katayama T, Yamamoto K et al. Physiology of consumption of human milk oligosaccharides by infant gut-associated bifidobacteria. J. Biol. Chem. 2011;286(40): 34583-34592.

28. Wang M, Li M, Wu S, Lebrilla CB, Chapkin RS, Ivanov I, Donovan SM. Fecal Microbiota Composition of Breast-Fed Infants Is Correlated With Human Milk Oligosaccharides Consumed. J. Pediatr. Gastroenterol. Nutr. 2015;60(6): 825-833.

29. Rasmussen SO, Martin L, Østergaard MV, Rudloff S, Roggenbuck M, Nguyen DN et al. Human milk 
oligosaccharide effects on intestinal function and inflammation after preterm birth in pigs. J. Nutr. Biochem. 2017;40: 141-154.

30. Drago L, Toscano M, De Grandi R, Grossi E, Padovani EM, Peroni DG. Microbiota network and mathematic microbe mutualism in colostrum and mature milk collected in two different geographic areas: Italy versus Burundi. 2017;11(4): 875-884.

31. Doran KS, Banerjee A, Disson O, Lecuit M. Concepts and mechanisms: Crossing host barriers. Cold Spring Harb. Perspect. Med. 2013;3: 1-20.

32. Hullar MAJ, Fu BC. Diet, the gut microbiome, and epigenetics. Cancer J. 2014;20: 170-175.

33. De Filippo C, Cavalieri D, Di Paola M, Ramazzotti M, Poullet JB, Massart S et al. Impact of diet in shaping gut microbiota revealed by a comparative study in children from Europe and rural Africa. Proc. Natl. Acad. Sci. U. S. A. 2010;107: 14691-14696.

34. Strati F, Di Paola M, Stefanini I, Albanese D, Rizzetto L, Lionetti P et al. Age and gender affect the composition of fungal population of the human gastrointestinal tract. Front. Microbiol. 2016;7: 1-16.

35. Lozupone CA, Stombaugh JI, Gordon JI, Jansson JK, Knight R. Diversity, stability and resilience of the human gut microbiota. Nature. 2012;489: 220-230.

36. Aagaard K, Petrosino J, Keitel W, Watson M, Katancik J, Garcia N et al. The Human Microbiome Project strategy for comprehensive sampling of the human microbiome and why it matters. FASEB J. 2013;27: 1012-1022.

37. Ma J, Coarfa C, Qin X, Bonnen PE, Milosavljevic A, Versalovic J, Aagaard K. mtDNA haplogroup and single nucleotide polymorphisms structure human microbiome communities. BMC Genomics. 2014;15: 257.

38. Clemente JC, Ursell LK, Parfrey LW, Knight R. The impact of the gut microbiota on human health: An integrative view. Cell. 2012;148: 1258-1270.

39. Peterson CT, Sharma V, Elmén L, Peterson SN. Immune homeostasis, dysbiosis and therapeutic modulation of the gut microbiota. Clin. Exp. Immunol. 2015; 179: 363-377.

40. Mueller NT, Bakacs E, Combellick J, Grigoryan Z, Dominguez-Bello MG. The infant microbiome development: mom matters. Trends Mol Med. 2015;21: 109-117.

41. Lagier JC, Khelaifia S, Alou MT, Ndongo S, Dione N, Hugon P et al. Culture of previously uncultured members of the human gut microbiota by culturomics. Nat Microbiol. 2016;1: 16203.

42. Eberl G. A new vision of immunity: homeostasis of the superorganism. Mucosal Immunol. 2010;3(5): $450-60$. 
43. Sleator RD. The human superorganism - Of microbes and men. Med. Hypotheses. 2010;74: 214215.

44. Goodacre R. Metabolomics of a superorganism. J. Nutr. 2007;137: 259S-266S.

44. Greuter D, Loy A, Horn M, Rattei T. ProbeBase-an online resource for rRNA-targeted oligonucleotide probes and primers: New features 2016. Nucleic Acids Res. 2016;44: D586-D589.

46. Gey A, Werckenthin C, Poppert S, Straubinger RK. Identification of pathogens in mastitis milk samples with fluorescent in situ hybridization. J. Vet. Diagn. Invest.2013; 25: 386-394.

47. Berry D, Schwab C, Milinovich G, Reichert J, Ben Mahfoudh K, Decker Tet al. Phylotype-level 16S rRNA analysis reveals new bacterial indicators of health state in acute murine colitis. ISME J. 2012;6(11): 2091-2106.

48. Borghi E, Borgo F, Severgnini M, Savini MN, Casiraghi MC, Vignoli A. Rett Syndrome: A Focus on Gut Microbiota. Int. J. Mol. Sci. 2017;18(2): pii: E344.

49. Masella AP, Bartram AK, Truszkowski JM, Brown DG, Neufeld JD. PANDAseq: paired-end assembler for illumina sequences. BMC Bioinformatics. 2012;13: 31.

50. Caporaso JG, Kuczynski J, Stombaugh J, Bittinger K, Bushman FD, Costello EK et al. QIIME allows analysis of high-throughput community sequencing data. Nat. Methods. 2010;7(5): 335-336.

51. Lozupone C, Knight R. UniFrac: A new phylogenetic method for comparing microbial communities. Appl. Environ. Microbiol. 2005;71: 8228-8235.

\section{FIGURE LEGENDS}

\section{Figure 1- Pioneer microbiota in the developing rodent gut}

Bar charts representing the relative abundance of 5 foetal intestines (I1-I5), amniotic fluids (A1A5) and placentas (P1-P5). The figure shows relative abundance of bacterial (A) phyla and (B) families.

Figure 2- Microbial biodiversity ( $\alpha$-diversity) is foetus-specific

(A) $\alpha$-diversity rarefaction curves according to Faith's phylogenetic distance ("PD whole tree"). $\mathrm{X}$-axis reports the number of sequences per sample, whereas $\mathrm{Y}$-axis shows the value of the metric. Samples are grouped based on foetus number. (B) Distribution of distances between $\alpha-$ diversity PD whole tree values; distances are labelled as "intra-" or "inter-category" according to 
foetus number. Dashed black line represents the mean of the distances, whereas the solid black line represents the median.

Figure 3- Microbiota composition ( $\beta$-diversity) is foetus-specific

(A) Principal coordinates analysis (PCoA) of the unweighted Unifrac distances; PCoA components 1 and 3 are reported. Samples are connected together on the basis of foetus number. (B) Boxplots of intra- and inter-category unweighted Unifrac distances among samples; categories are based on the foetus number. (C) Boxplots of intra-category weighted Unifrac distances among samples; samples are grouped according to foetus, tissue or dam.

\section{Figure 4- Eubacteria in the developing rodent gut lumen}

Confocal microscopy images showing (A) eubacteria (in green) in the lumen of a 16 days post coitum rat foetus; $(B)$ at a higher magnification (inset is represented as a white box in $A$ ) note the typical bacterial morphology and it is possible to identify few Bacteroides spp. (yellow). In blue is DAPI (nuclei) and $50 \mu \mathrm{m}$ scale bar is reported in B. Asterisks indicate bacterial cells.

\section{Figure 5- Eubacteria in the developing human gut}

(A) Bar charts representing the family relative abundance at family level of three foetal human intestines (29, 31, and 33 weeks of gestation, respectively). (B) Representative confocal microscopy images of in situ hybridisation showing the presence of eubacteria (in green-, inset showing higher magnification) in the lumen human foetuses. In blue is DAPI (nuclei) and $100 \mu \mathrm{m}$ and $50 \mu \mathrm{m}$ (inset) scale bars are reported. Asterisks indicate bacterial cells. 


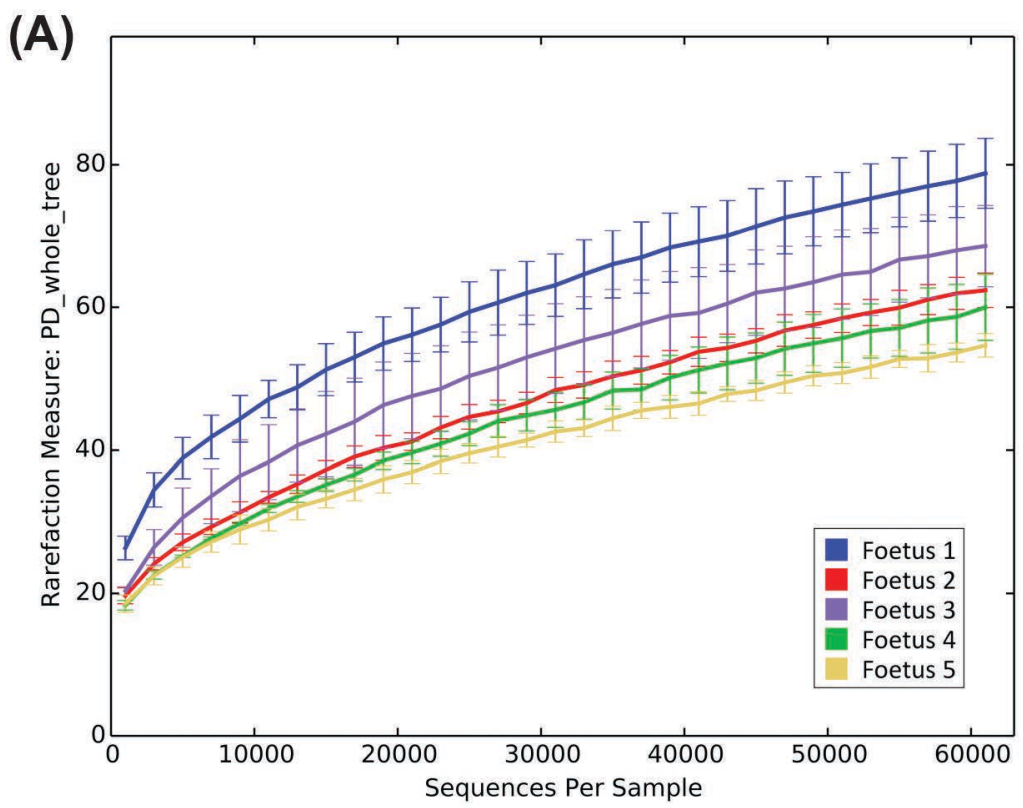

(B) 35

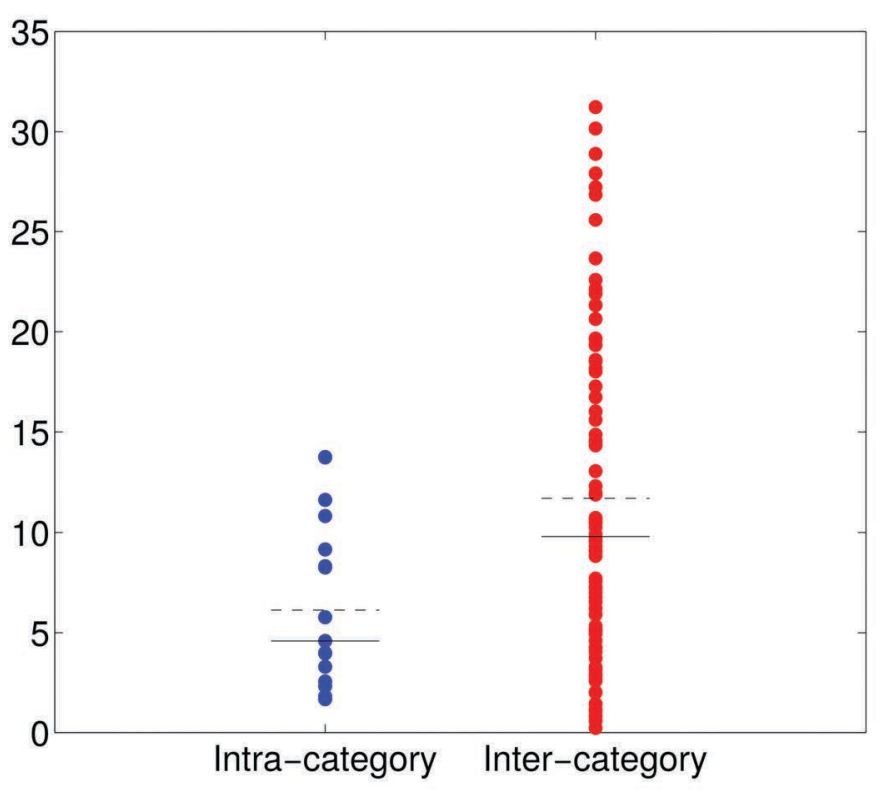


$\mathbf{A}$

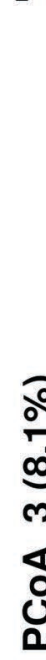

1
0
0
0
0
0
0
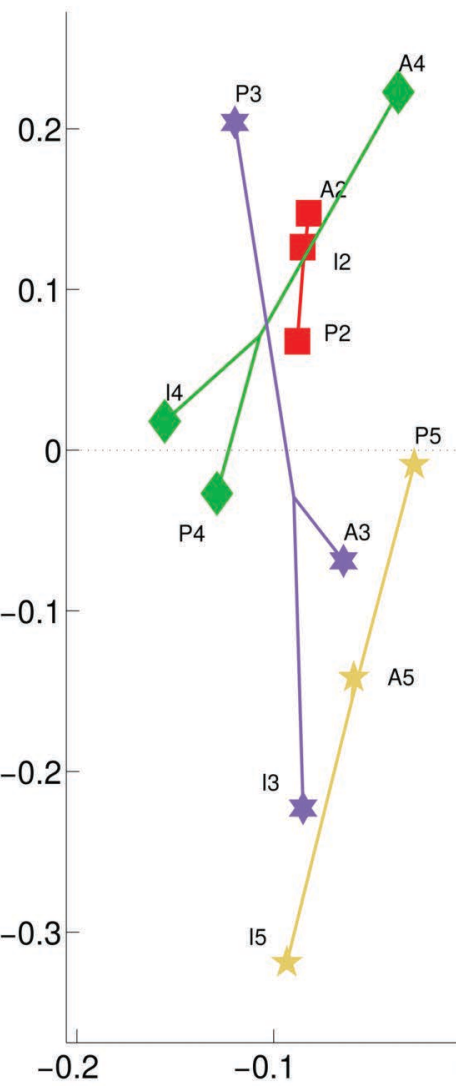

$-0.1$

0.1

0.2

PCoA_1 (12.3\%)

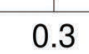

Foetus 1

Foetus 2

Foetus 3

Foetus 4

Foetus 5

B

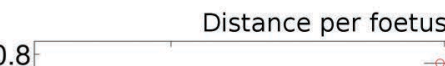

.78

0.76

0.74

0.72

0.7
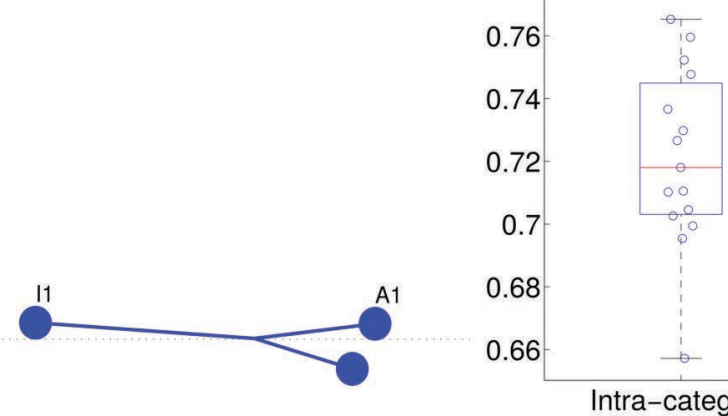

0.68

0.66

P1

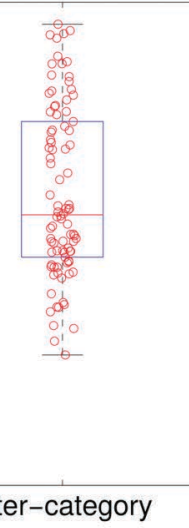

C

Intra-category Inter-category

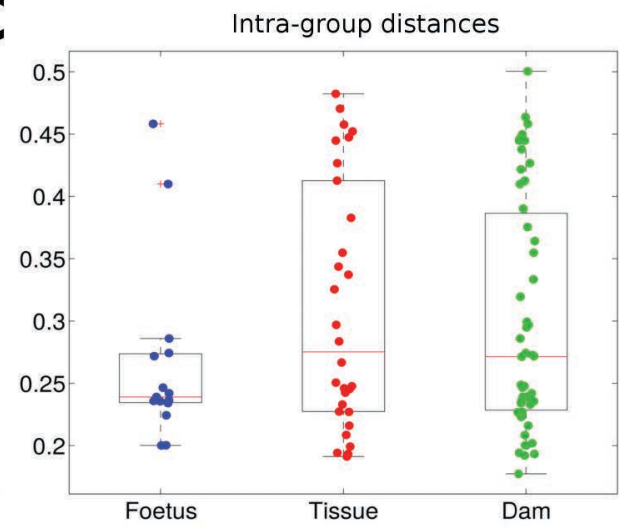


bioRxiv preprint doi: https://doi.org/10.1101/236190; this version posted December 18,2017 . The copyright holder for this preprint (which
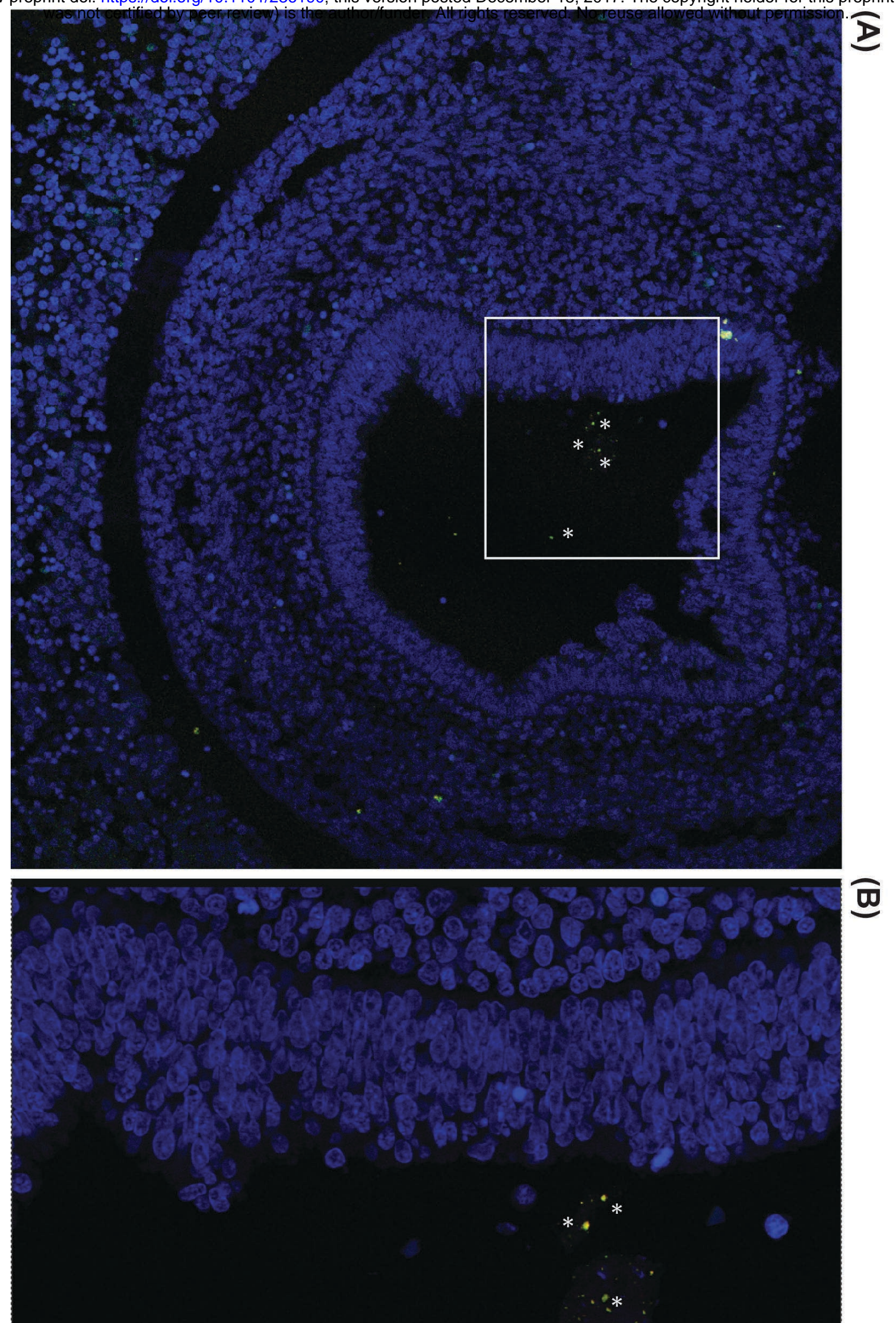
bioRxiv preprint doi: https://doi.org/10.1101/236190; this version posted December 18,2017 . The copyright holder for this preprint (which was not certified by peer review) is the author/funder. All rights reserved. No reuse allowed without permission.

\&

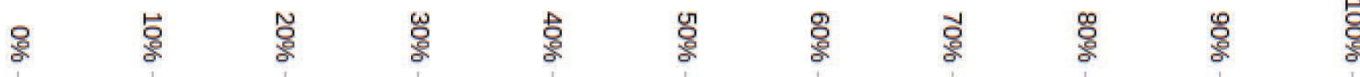

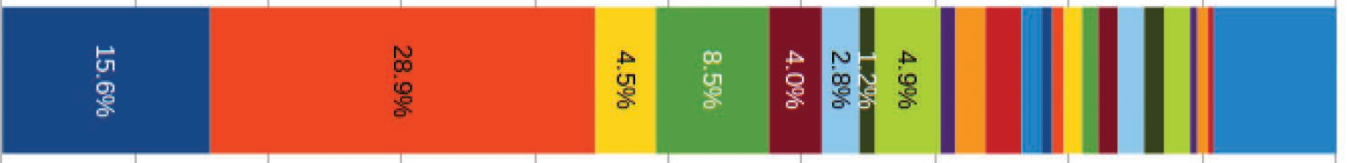

$\stackrel{\omega}{\varphi}$

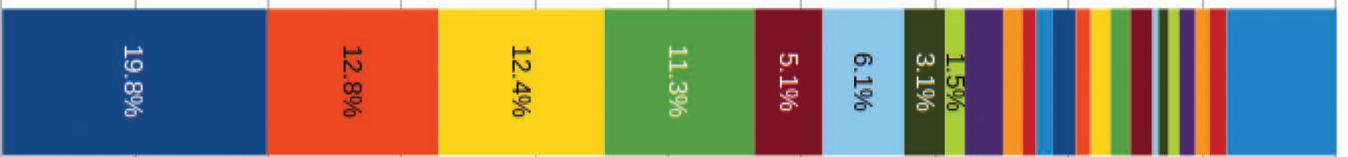

$\omega$
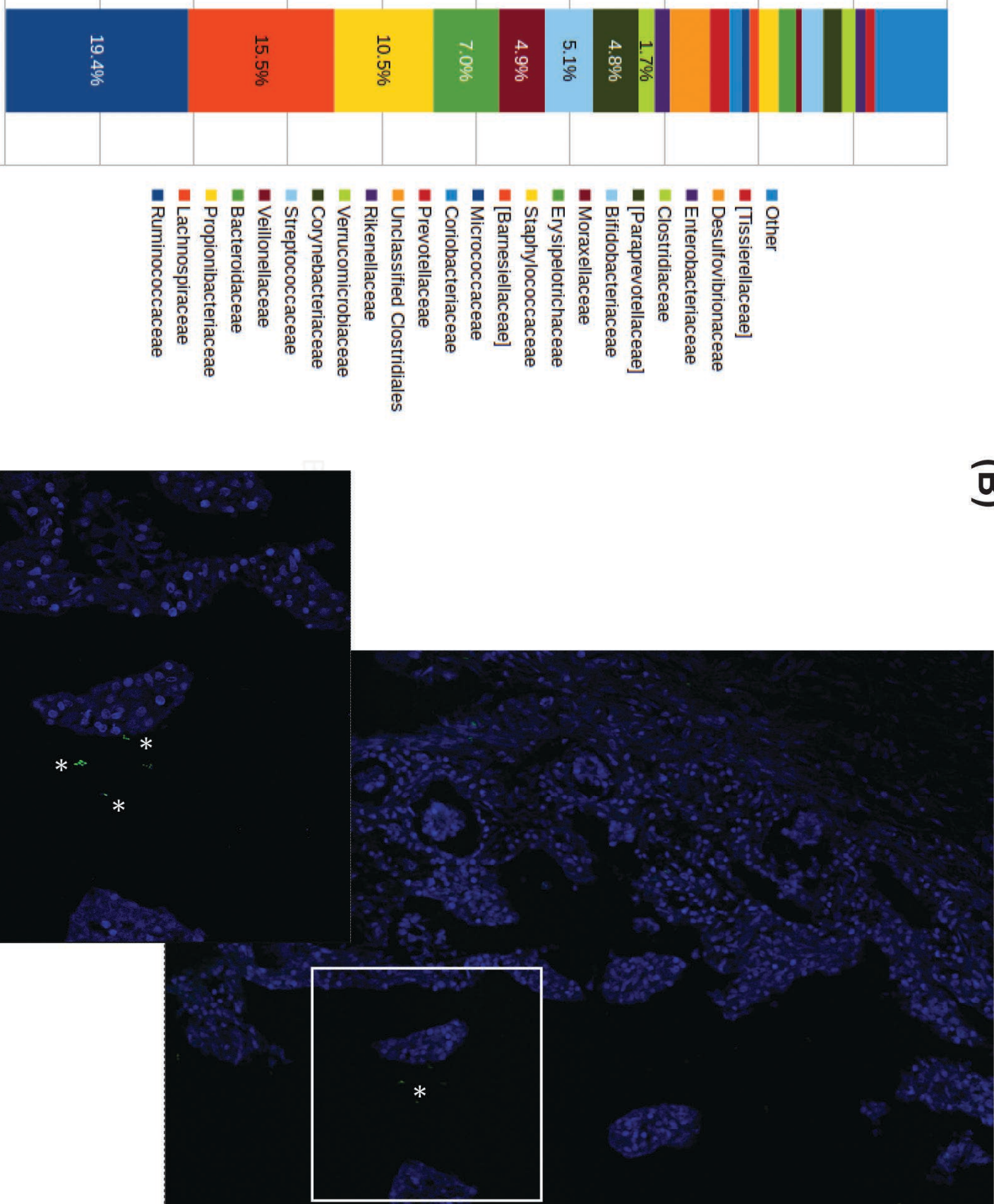